\title{
THE AMORPHOUS SUBSTANCE IN THE TRABECULAR MESHWORK*
}

BY

\author{
F. VRABEC
}

From the Pharmacological Institute, University of Uppsala, Sweden, and the First Eye Clinic, University of Prague, Czechoslovakia.

VIRCHOW (1910), in his description of the micro-anatomy of the trabecular meshwork, mentioned the presence of some unfamiliar tissue-element in addition to the known structures. In the trabecular meshwork of the horse he observed fine threads originating and terminating in the endothelial cells, and running through the empty spaces of the meshwork. I have found no other description of this substance in the subsequent literature on the morphology of the trabecular system.

In working on the problem of the innervation of the trabecular meshwork (Vrabec, 1954), I have very often noted a similar picture. As my sections were mainly cut on the freezing-microtome, the conservation of the tissue components was better than in paraffin sections. In flat preparations of the trabecular meshwork, thin sheets of a perfectly transparent substance were very often seen. Their existence was indicated only by the presence of silver deposits. Even in frozen sections, however, it was not possible to explore this substance in detail, as it was not found in meridional sections. A systematic search for a better method of embedding was therefore made. Paraffin infiltration causes great deformation and celloidin was also found to be deleterious to this delicate tissue component. Many other methods were tested with little success. At the instigation of Prof. J. Wolf of Prague, Celodal-embedding was then tried. This method was introduced by Hoepke (1939), and is described in the text-book of microscopical technique by Romeis (1943). It causes only minimal changes in the amorphous substance, but the main disadvantage of the method is that once it is polymerized Celodal cannot be removed from the sections by any solvent, and it takes up some colour.

\section{Material and Methods}

Sheep's eyes were dissected equatorially, and fixed in 10 per cent. formol for a week. The anterior segment was then rinsed for $12 \mathrm{hrs}$ in tap water, and the lens removed care-

* Received for publication October 26, 1956. 
fully from behind. Next the anterior segment was dehydrated for $24 \mathrm{hrs}$ in 50 per cent. ethanol, and after dehydration it was divided into four quadrants. One quadrant was embedded in paraffin as a control; the other three were placed in a mixture of $50 \mathrm{ml}$. 70 per cent. ethanol, $50 \mathrm{ml}$. Celodal*, $5 \mathrm{ml} .40$ per cent. formol, and $1 \mathrm{ml} .25$ per cent. $\mathrm{w} / \mathrm{v}$ aqueous solution of ammonium chloride. The pieces remained in this mixture for 2 days and were then transferred to the definitive embedding mixture of $100 \mathrm{ml}$. Celodal, $5 \mathrm{ml} .40$ per cent. formol, and $2 \mathrm{ml}$. ammonium chloride solution. This mixture was allowed to polymerize in a Petri dish for. 2 days. After this time, it was firm enough to allow blocks containing the pieces of tissue to be cut. Polymerized Celodal is transparent, and exact orientation of the pieces is very easy. The blocks were then fixed on wooden supports with a few drops of a mixture of Celodal and ammonium chloride, and allowed to remain at room temperature. In a few days they had hardened to a suitable consistency. Such blocks can be cut in a similar manner to celloidin blocks, using an oblique knife, and the sections can be removed and stored in 60 per cent. ethanol.

Our sections were $15 \mu$ thick, but $10 \mu$ sections can easily be cut from such material.

Staining.-1 or $2 \mathrm{hrs}$ in a 1:1000 solution of cresyl violet (Grübler-Hollborn) in distilled water. The embedding medium is only slightly stained.

Mounting.-Permanent preparations are difficult to make by this method, because mounting in Canada balsam causes the metachromasia to disappear, and the colour substance soon diffuses out of the section into the mounting medium. The use of glycerol gelatin conserves the metachromasia, but even such preparations deteriorate in a few months, and the endothelial cells and their nuclei deprive the amorphous substance of dye.

\section{Findings}

In the sheep's eye all the spaces between the trabeculae seemed to be filled by an amorphous substance staining a reddish violet. In the meshes of the uveal trabeculae no appreciable amount of this substance was found. Although the substance did without doubt stain metachromatically, its colour was quite different from the very reddish metachromasia of the corneal stroma (Fig. 1, overleaf). The collagen bundles of the sclera stained faintly pink, but the trabeculae appeared unstained probably by contrast with the compact coloured substance (Fig. 2, overleaf). The ciliary muscle following the trabecular meshwork took on an intensive, more bluish tone. The endothelial cells were practically invisible against the great amount of amorphous substance, but in thin sections or older preparations, they could be seen as a very thin, darkly-coloured lining of the colourless trabeculae.

We compared Celodal and paraffin sections, stained with cresyl violet and mounted in glycerol gelatin. The result is clearly shown in Figs 3 and 4 (overleaf). After embedding in paraffin a considerable shrinkage of the amorphous substance takes place, resulting in the formation of empty spaces. The endothelial cells remain close to the trabeculae, and only very fine threads run between the distorted layers of tissue. The concentration of amorphous substance is highest at the tip of the trabecular meshwork close to the cornea. The next highest concentration is in the external layers of the meshwork close to the inner surface of the sclera. The innermost layer of

\footnotetext{
* Celodal, a product of Bayer, Leverkusen, is a clear, thick liquid which polymerizes after the addition of acidic
} substances. All the instruments and dishes must be rinsed immediately after use. 


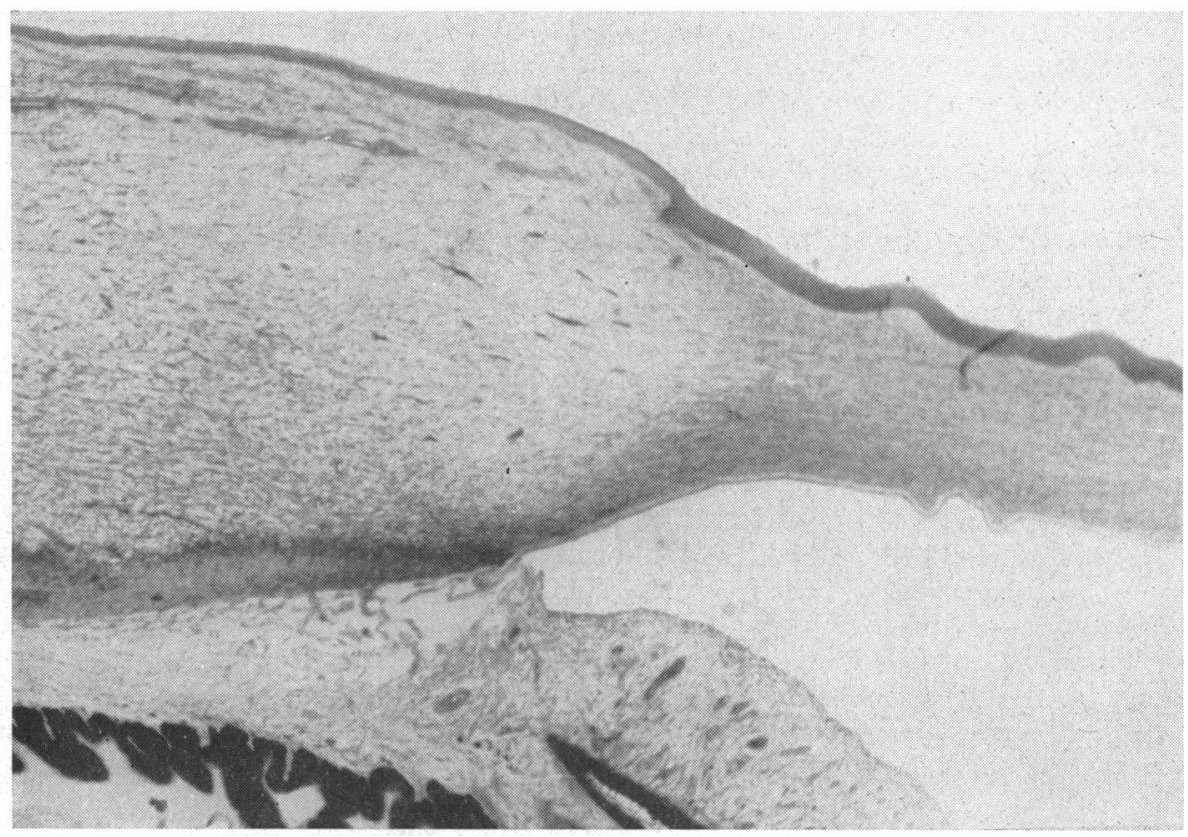

FIG. 1.-Meridional section of the chamber angle of the sheep's eye. Celodal embedding, cresyl violet stain. Metachromasia of the corneal stroma, intensive staining of the scleral meshwork, and very faintly coloured sclera. The uveal meshwork does not contain any coloured substance. $\times 50$.

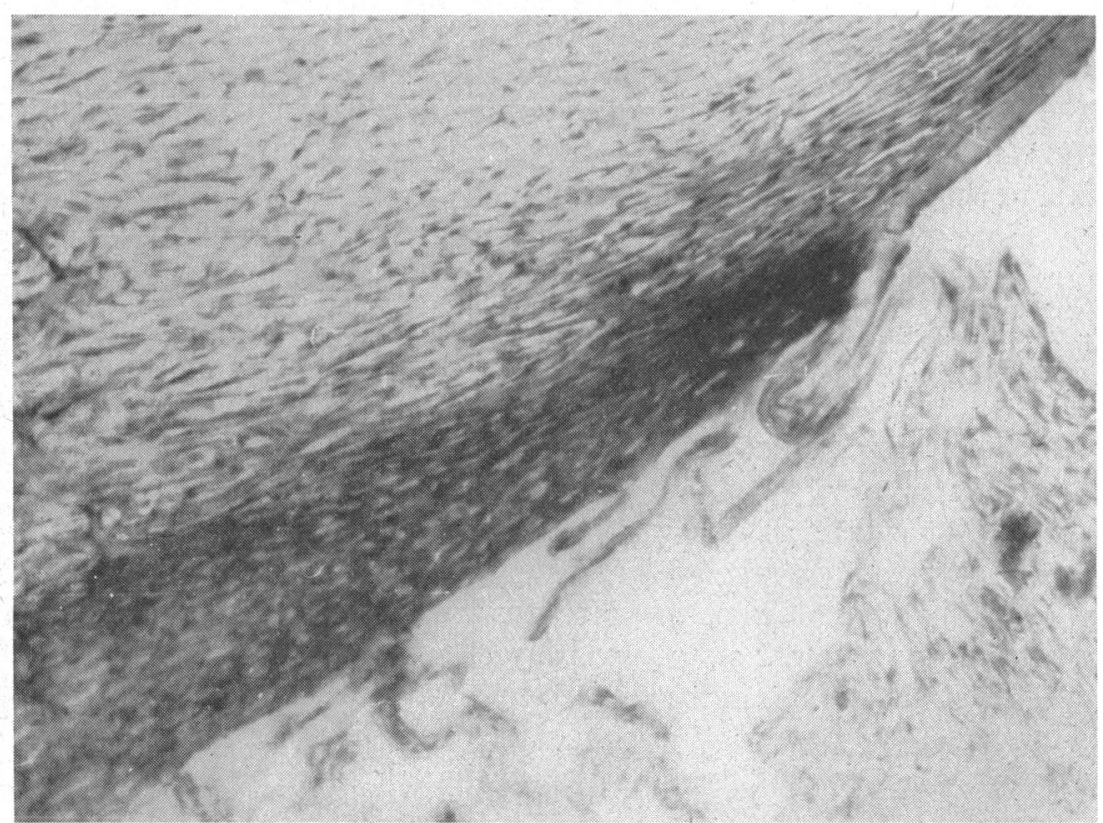

Fig. 2. - Same section $\times 200$. The scleral trabecular meshwork appears strongly stained. 


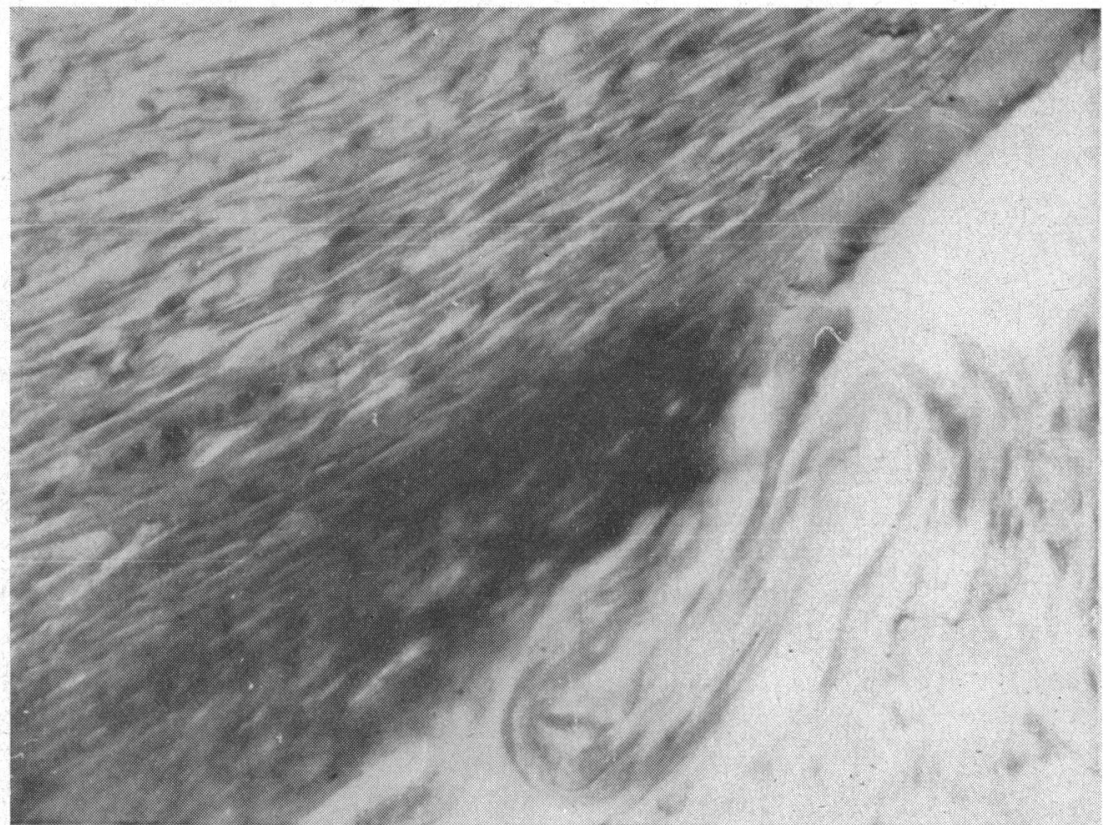

Fig. 3.-Detail of tip of scleral meshwork of same section, showing great accumulation of amorphous substance in this region. $\times 400$.

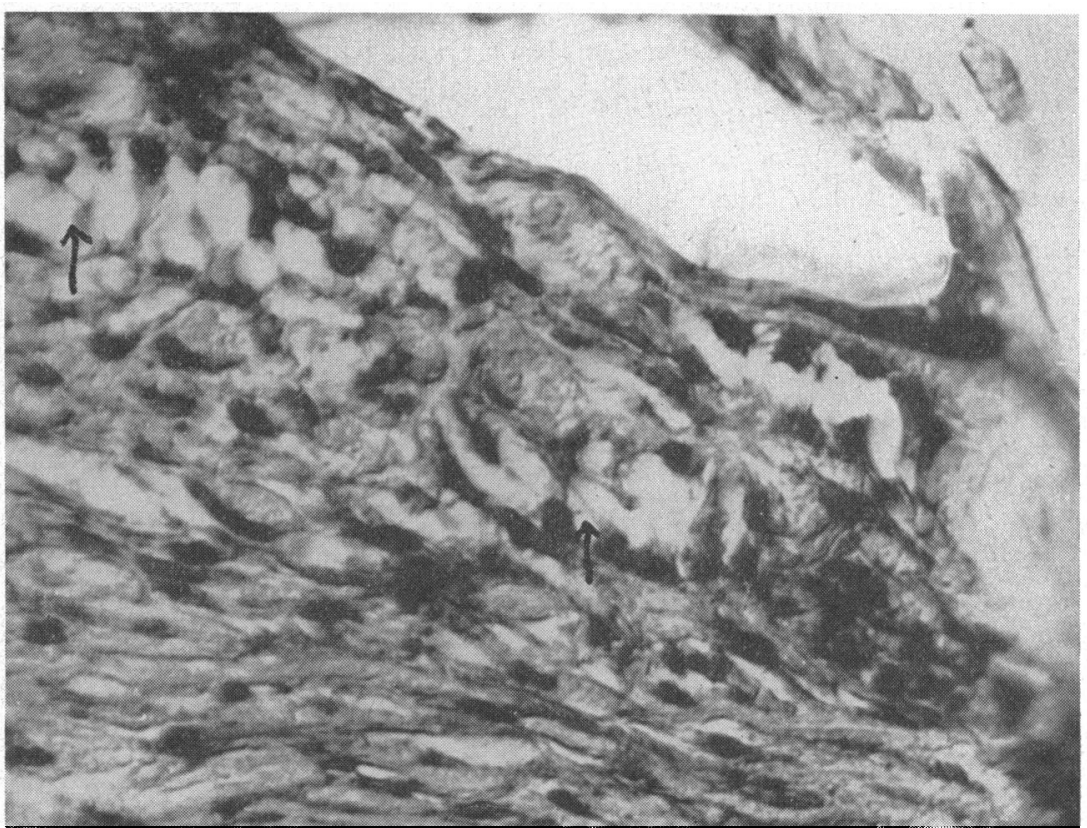

FIG. 4.-Same region of scleral meshwork of same eye after paraffin embedding. All other staining and mounting manipulations were identical with those used in Fig. 3. The amorphous substance appears to have shrunken to fine threads, some of which are visible near arrows. $\times 400$ 
scleral trabeculae come next, and the middle layers of the meshwork are the palest.

The metachromatic material can also be seen in non-embedded preparations. On staining (with a 1:1000 solution of toluidin blue in water) a very thin, teased-out strip of the scleral meshwork of the formol-fixed cow's eye a very distinct metachromasia of the meshwork can be seen.

\section{Discussion}

The anatomical demonstration of the existence of an amorphous substance in the spaces of the trabecular meshwork confirms the physiological evidence provided by the work of Bárány (1956). It should be mentioned, however, that the human trabecular. meshwork has been seen to stain metachromatically by Persson (1954) and by Berggren (1956), but these authors were not able to study the actual localization of the metachromatic material since they used only the classical embedding media.

\section{REFERENCES}

BáRÁnY, E. (1956). "First Conference on Glaucoma". Josiah Macy Jr. Foundation, New York.

BergGren, L. (1956). Personal communication.

HOEPKE, H. (1940). Z $Z$. wiss. Mikr., 56, 453

Persson, B. (1954). Acta Soc. Med. Upsalien., 59, 260. Quoted by Bárány (1956).

RomeIS, B. (1943). "Taschenbuch der mikroskopischen Tecknik", 14th ed., p. 157. Oldenbourg, Berlin.

VIRCHOW, H. von (1910). "Graefe-Saemisch Handbuch der gesamten Augenheilkunde", 2nd ed., Bd. 1, Abt. 1, p. 280. Englemann, Leipzig.

VRABEC, F. (1954). Ophthalmologica (Basel), 128, 359. 